\title{
Prevalencia de síntomas de rinitis alérgica y su relación con factores de riesgo en escolares de Santiago, Chile
}

\author{
Solange Caussade L1, G onzalo Valdivia $C^{2}$, \\ Héctor $N$ avarro $M^{1}$, Enrique Pérez $B^{a}$, \\ Andrés Aquevedo $S^{a}$, Ignacio Sánchez $D^{1}$. \\ Risk factors and prevalence \\ of allergic rhinitis among \\ Chilean children
}

Background: The prevalence of asthma and allergic rhinitis experienced a steady increase in the last years, probably associated to changes in lifestyles. Aim: To assess the prevalence of allergic rhinitis, to evaluate changes over time (1994-2000), and to describe risk factors. Material and Methods: The International Study of Asthma and Allergies in Childhood (ISAAC) core questionnaire, with questions added about socioeconomic status (SES), was applied to 4594 children between 6-7 years old and 13-14 years old during october-december 2000. Attendance to public or private schools was also used a SES proxy. The results were compared with those of a similar survey in 5281 children, performed in 1994. Rhinitis symptoms (ever) (SR), rhinitis symptoms within last 12 months (SR12) and medical diagnosis of rhinitis (DR) were assessed. Results: There was a significant increase in the prevalence of SR, SR 12 and DR in both age groups in 2000, compared to 1994. SR older children showed a higher prevalence of SR compared with the youngest group $(\mathrm{p}=0.003)$. No age differences were observed in the prevalence of SR12 and DR. Both SR and SR12 were more prevalent at schools of medium and low-medium SES ( $p=0.003$ and $p=0.002$ respectively). DR was significantly more prevalent among children of high SES. A better mother educational level was associated to higher prevalence of SR and SR12 ( $p=0.03$ and $p=0.04$ ). Father educational level was associated to DR ( $p=0.007)$. The prevalence of SR12 was higher in households with carpets $(\mathrm{p}=0.017)$. The prevalence of $\mathrm{DR}$ was higher in houses with smokers $(\mathrm{p}=0.03)$ and gas heating ( $p=0.005)$. None of the three variables were related to gender. Conclusions: The prevalence of SR, SR12 and DR increased significantly in a short time period (6 years). Our results support a positive association between DRand high SES (Rev Méd Chile 2006; 134: 456-64).

(Key words: Pediatrics; Rhinitis, allergic, perennial; Rhinitis, allergic, seasonal)

Recibido el 12 de agosto, 2004. Aceptado el 17 de octubre, 2005.

${ }^{1}$ Sección Respiratorio Pediátrico, Pontificia Universidad Católica de Chile. ${ }^{2}$ Departamento Salud Pública, Pontificia Universidad Católica de Chile.

aAyudante Alumno Departamento Salud Pública, Pontificia Universidad Católica de Chile.

Correspondencia a: Dra Solange Caussade. Sección Respiratorio Pediátrico. Lira 85 - 5 Piso, Santiago. Fono: 3543767.

Fax: 2473879. E mail: mcaussa@med.puc.cl 
$\mathrm{L}$ a rinitis alérgica es la condición crónica más común en la población adulta y pediátrica, lo que determina un impacto personal, social y económico muy importante ${ }^{1,2}$. La etiología de la rinitis es diversa, siendo la alergia su principal factor desencadenante, determinada especialmente por reacciones de hipersensibilidad mediada por inmunoglobulina E. Otras causas de rinitis incluyen factores no alérgicos, irritantes, infecciones, trastornos hormonales y ocupacionales ${ }^{2-4}$.

El Estudio Internacional de Asma y Alergias en la Infancia (ISAAC) fue diseñado para adquirir información acerca de la prevalencia de asma, rinitis y eczema en la niñez, mediante una metodología estandarizada que permite la comparación internacional. Este estudio mostró variaciones importantes entre los 56 países participantes ${ }^{5}$. La prevalencia de síntomas de rinoconjuntivitis durante los 12 meses previos a la aplicación del cuestionario, varió entre 0,8 y $14,9 \%$ en el grupo de 6-7 años y entre 1,4 y 39,7\% en el de 13-14 años. En relación al diagnóstico de asma, en el grupo de 6-7 años, la prevalencia fluctuó entre 1,0 y $30,3 \%$ y en el grupo de $13-14$ años entre 1,6 y 30,4\%6-8. En los 5 centros estudiados en Chile, presentaban diagnóstico de asma 9,7 a 16,5\% de niños de 6-7 años y 7,3 a 12,4\% del grupo de 13-14 años ${ }^{9}$. En el estudio internacional, se encontró una asociación significativa entre asma y rinitis: en países con baja prevalencia de asma (menor de 5\%), la prevalencia de rinitis también fue baja, en los países con alta prevalencia de asma (mayor de 30\%), la prevalencia de rinitis fue alta $(15-20 \%)^{7}$. En Santiago, año
2000, se encontró la presencia de asma en $26,5 \%$ de los niños portadores de rinitis ${ }^{10}$.

La prevalencia de asma y rinitis alérgica ha presentado un aumento progresivo en los últimos años, lo que se ha atribuido a diversos factores relacionados con el estilo de vida occidental como cambios en la dieta, mayor permanencia en espacios cerrados, exposición a ciertos alergenos y mejor nivel socioeconómico ${ }^{11-19}$.

Los objetivos de esta investigación fueron determinar la prevalencia de algunos síntomas seleccionados de rinitis alérgica en el año 2000, compararla con las cifras encontradas en 1994 (Estudio ISAAC, sector Santiago Centro) ${ }^{9}$, y describir la influencia del nivel socioeconómico y factores de riesgo ambientales en la forma de presentación de la enfermedad.

\section{PACIENTES Y MÉTODO}

Se estudiaron escolares de 6-7 años y 13-14 años de ambos sexos, los cuales asistían regularmente a establecimientos educacionales de las áreas Santiago Oriente $(\mathrm{n}=2.866,16$ colegios) y Santiago Centro ( $n=1.728 ; 11$ colegios), seleccionados por muestreo aleatorio, estratificado según tipo de establecimiento, a partir del directorio elaborado por el Ministerio de Educación. Durante los meses de noviembre y diciembre del año 2000, se aplicó el cuestionario ISAAC, el cual hace énfasis en relatar síntomas de rinitis no relacionados con el resfrío común ni gripe ${ }^{4}$ (Tabla 1). Se agregaron

\section{Tabla 1. Cuestionario para niños de 6-7 años y 13-14 años. Síntomas de rinitis alérgica}

1. Alguna vez en su vida tha tenido su hijo (a) problemas de estornudos, romadizo, o se le tapa la nariz?*

2. En los últimos 12 meses, ctuvo su hijo (a) problemas de estornudos, romadizo, o se le tapa la nariz?*

3. En los últimos 12 meses, junto con el problema de la nariz ile picaban y le lloraban los ojos?

4. ¿En cuál de los 12 meses ocurrían estos problemas nasales?

5. En los últimos 12 meses ¿en qué cantidad interfirieron estos problemas nasales con sus actividades diarias?

6. ¿Ha tenido alguna vez su hijo (a) Rinitis Alérgica?

*Síntomas no relacionados con gripe o resfrío. 
indicadores de nivel socioeconómico y contaminación intradomiciliaria (Tabla 2). El cuestionario fue distribuido en la sala de clases, junto a una nota explicativa y solicitud de consentimiento informado para los padres, previo contacto con profesores, explicando la metodología y procedimientos del estudio. Este fue respondido por los padres de los escolares de 6-7 años, y por los escolares de 13-14 años, en conjunto con sus padres. Los cuestionarios fueron recuperados en dos fechas, para optimizar la tasa de respuesta. La metodología de aplicación de este instrumento siguió un procedimiento similar al reportado en el estudio chileno ISAAC ${ }^{9}$.

Fueron seleccionadas tres variables relacionadas con el componente de rinitis del estudio ISAAC, incluyendo una pregunta de carácter general y dos de mayor grado de especificidad. Las variables estudiadas fueron: síntomas de rinitis alérgica alguna vez en la vida (SR), síntomas de rinitis alérgica los últimos 12 meses, en ausencia de resfrío (SR12) y diagnóstico de rinitis alérgica (DR) (preguntas № 1, 2 y 6, Tabla 1). Se asume que la pregunta № 6 implica diagnóstico médico de rinitis alérgica. Se analiza en cada una de ellas su relación con edad, sexo, contaminantes y alergenos intradomiciliarios (tipo de combustible utilizado para calefacción y cocinar, material que cubre el piso de la casa, mascotas en el hogar, exposición a humo de tabaco) y nivel socioeconómico. Este último se definió en base a la ubicación del establecimiento: Santiago Centro corresponde a nivel medio y medio-bajo (establecimientos públicos y subvencionados) y Santiago Oriente a nivel acomodado (colegios particulares). Estas variables se relacionaron, además, con el nivel educacional de los padres, clasificados como bajo (sin educación o educación básica), medio (educación media completa) y superior (educación técnica profesional o universitaria). Se contó con los datos de 5.281 encuestas realizadas entre agosto y octubre del año 1994 en el sector Santiago Centro, en diferentes establecimientos que los de este estudio (2.327 de 6-7 años y 2.954 de 13-14 años). Este cuestionario incluía los antecedentes respecto a síntomas de asma, rinitis y eczema, no contemplando la evaluación del NSE. El estudio fue aprobado por el Comité de Ética de la Escuela de Medicina de la Pontificia Universidad Católica de Chile.

Estadística. Los datos recolectados se ingresaron a una base de datos Epi-Info. El análisis estadístico se realizó mediante comparación de proporciones, regresión logística condicional, tablas de contingencia y chi cuadrado para tendencias lineales según correspondiera. Se calcularon odds ratio de prevalencia crudos para las variables relacionadas con rinitis y en una segunda instancia odds ratios ajustados por un conjunto de potenciales variables confundentes. Para el análisis comparativo de las prevalencias a estudiar según su fluctuación en el período 1994-2000, al igual que las comparaciones según grupo etáreo, el tamaño de la muestra

\section{Tabla 2. Contaminación intradomiciliaria y nivel socioeconómico}

1. ¿Cuál es el principal tipo de combustible utilizado para calefacción de su casa?*

2. ¿Cuál es el principal combustible que usa para cocinar en su casa?*

3. ¿Cuál es el material que cubre el piso de su casa?*

4. ¿Tiene usted animales (mascotas) en su casa?¿Cuál (es)?

5. ¿Fuma alguien en su casa actualmente? ¿Cuántas personas?

6. Ubicación del colegio.

7. Madre, ¿completó usted su: Educación Básica?, Educación Media?, ¿Educación Técnico Profesional?, ¿Universitaria?**

8. Padre, ¿completó usted su: Educación Básica?, Educación Media?, ¿Educación Técnico Profesional?, ¿Universitaria?*k

*Debe anotarse el más importante según listado descrito luego de cada pregunta. ${ }^{*}$ Si no se ha completado, debe señalarse el último año al que asistió. 
permitió evaluar diferencias de hasta 5 puntos porcentuales, con 95\% de confianza y $80 \%$ de potencia. Los resultados se expresaron como promedios, error estándar e intervalos de confianza al $95 \%$. Se estableció como valor estadísticamente significativo un $\mathrm{p}<0,05$. En los análisis se utilizó el software STATA en versión 6.0.

\section{RESULTADOS}

Durante el año 2000, se distribuyeron 7.864 encuestas, recuperándose 6.000 (76,3\%). Se respondió en forma completa las preguntas relacionadas con síntomas de rinitis alérgica en 4.594 de ellas $(76,5 \%)$, las que fueron utilizadas para los análisis: 1.728 del área Santiago Centro y 2.866 del área oriente, 2.283 mujeres y 2.303 hombres (en 8 casos no se identificó sexo), 2.382 de 6-7 años y 2.212 de 13-14 años. Se contó con los datos de 5.281 encuestas del año 1994, realizadas solamente en el área central, 2.670 mujeres y 2.590 hombres (en 21 no se identificó sexo), 2.327 de 67 años y 2.954 de 13-14 años.

En el análisis del grupo total del año 2000 no se observó diferencia en las prevalencias de las tres variables según sexo: SR en $41,12 \%$ de mujeres y $40,83 \%$ de hombres ( $p=0,81$ ), SR12 en $37,01 \%$ de mujeres y $36,63 \%$ de hombres $(p=0,79)$ y DR en $19,49 \%$ de las mujeres y $21,67 \%$ de los hombres $(p=0,07)$, semejante a lo ocurrido el año $1994^{9}$. Al comparar por edad, sólo se detectó mayor prevalencia de SR en los escolares de 13-14 años con respecto a los de 6-7 años $(45,74 \%$ vs $40,46 \%, p=0,003)$. En los escolares de 13-14 años, la prevalencia de SR12 fue de 40,49\% y en los de 6-7 años de 37,1\% ( $p=0,055)$; en el grupo de 13-14 años, se encontró DR en 21,37\% y en el de 6-7 años 21,99\% ( $p=0,67)$ (Figura 1). En 2000, los escolares de establecimientos del sector centro mostraron mayor prevalencia de SR con respecto a los pertenecientes al sector oriente: $43,64 \%$ vs $39,24 \%(p=0,003)$, ocurriendo lo mismo en relación a SR12: $39,49 \%$ vs $35,04 \%$ $(p=0,002)$. Se observó lo inverso con respecto a DR: $23,13 \%$ en sector oriente, $17,03 \%$ en sector central ( $p<0,0001$ ) (Figura 2). Al analizar la influencia del nivel educacional de los padres, se encontró asociación entre mayor prevalencia de SR y mejor nivel educacional de la madre: $30,52 \%$ en madres con nivel educacional bajo, $25,91 \%$ nivel medio y $43,56 \%$ nivel superior $(\mathrm{p}=0,013)$; lo mismo se observó con respecto a SR12: 30,34\% cuando la madre tenía nivel educacional bajo, 25,91\% nivel medio y 43,74\% nivel superior $(p=0,04)$. El mejor nivel educacional del padre se asoció con mayor prevalencia de $\mathrm{DR}$ : $22,02 \%$ si el padre tenía nivel educacional bajo, $13,49 \%$ nivel medio y $64,49 \%$ nivel superior $(\mathrm{p}=0,007)$.

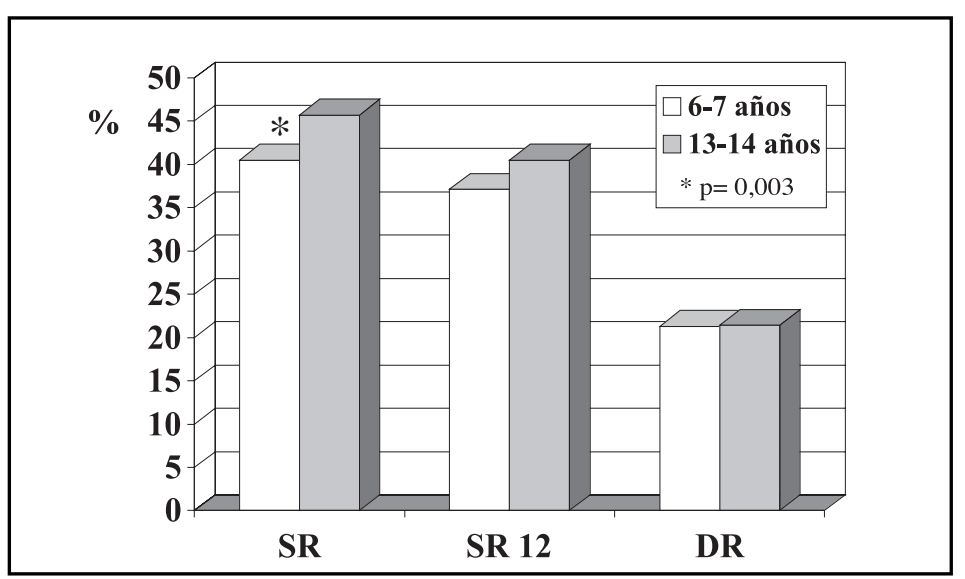

Figura 1. Prevalencias año 2000, áreas central y oriente, ambos sexos, ambos grupos etáreos (6-7 años $n=2.382$, 13-14 años $\mathrm{n}=2.214$ ) ( $\mathrm{SR}=$ síntomas de rinitis alérgica alguna vez en la vida; $\mathrm{SR} 12=$ síntomas de rinitis alérgica los últimos 12 meses; DR= diagnóstico médico de rinitis alérgica). 


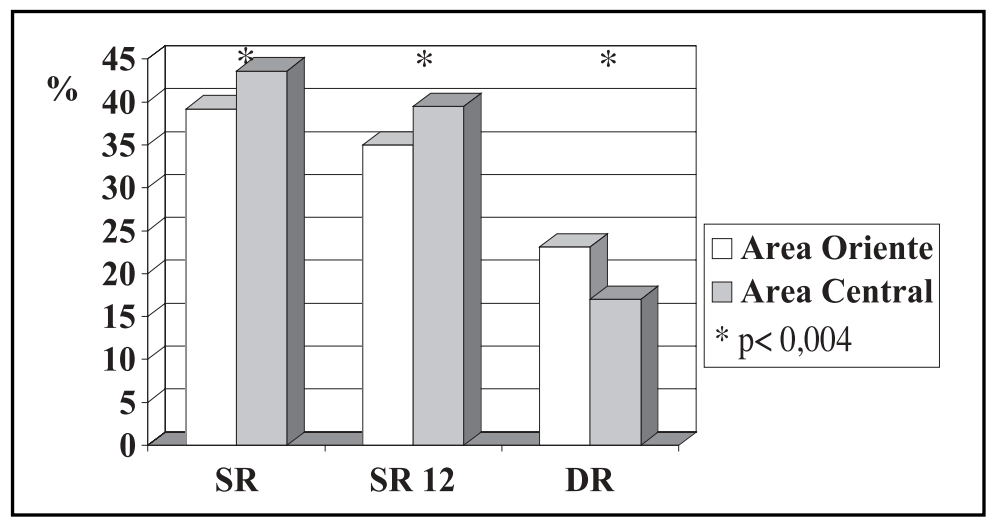

Figura 2. Prevalencias según nivel socioeconómico (NSE), año 2000, ambos sexos, ambos grupos etáreos (área oriente $\mathrm{n}=2.866$, área central $\mathrm{n}=1.728$ ) ( $\mathrm{SR}=$ síntomas de rinitis alérgica alguna vez en la vida; $\mathrm{SR} 12=$ síntomas de rinitis alérgica los últimos 12 meses; $\mathrm{DR}=$ diagnóstico médico de rinitis alérgica).

En relación con alergenos y contaminantes intradomiciliarios, se observó una mayor prevalencia de SR en hogares con cubrepiso o alfombra $(p=0,017)$ y mayor prevalencia de DR cuando había fumadores en el hogar $(p=0,003)$ y en aquellos hogares con calefacción a gas $(\mathrm{p}=0,005)$. No hubo diferencia significativa en la prevalencia de síntomas según tipo de combustible para cocinar y presencia de mascotas.

Al comparar los valores obtenidos entre los años 1994 y 2000 en el área central de Santiago, se observa un aumento significativo en la prevalencia de SR: $29,7 \%$ vs $40,46 \%$ en el grupo de 6-7 años y $35,1 \%$ vs $45,74 \%$ en el grupo $13-14$ años
( $p=0,0001$ en ambos) (Figura 3), SR 12: $27 \%$ vs $37,1 \%$ en el grupo de $6-7$ años y $27,9 \%$ vs $40,49 \%$ en el grupo de 13-14 años ( $p=0,0001$ en ambos) (Figura 4) y DR: $8,8 \%$ vs $21,99 \%$ en el grupo de 6 7 años y $10,8 \%$ vs $21,37 \%$ en el grupo de $13-14$ años ( $p=0,0001$ en ambos) (Figura 5).

\section{DisCUSIÓN}

Según esta investigación, se observa que en la ciudad de Santiago, al igual que en países desarrollados, la percepción de síntomas sugerentes de rinitis alérgica ha aumentado en forma considerable en un

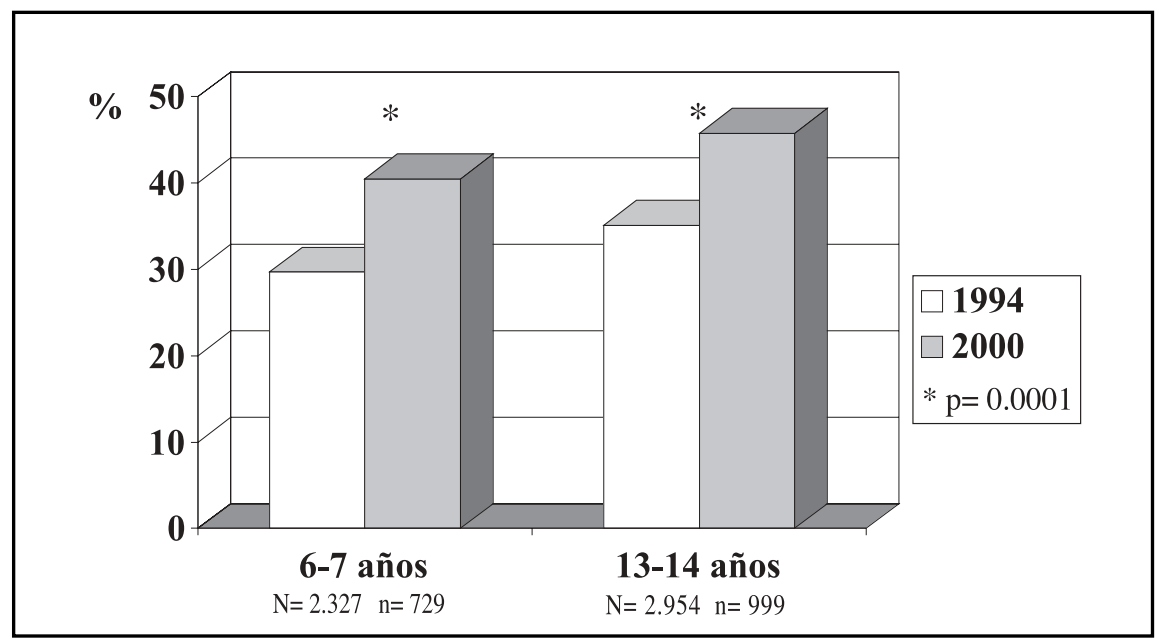

Figura 3. Síntomas de rinitis alérgica alguna vez en la vida (SR). Comparación 1994-2000, área central de Santiago, ambos grupos etáreos. 


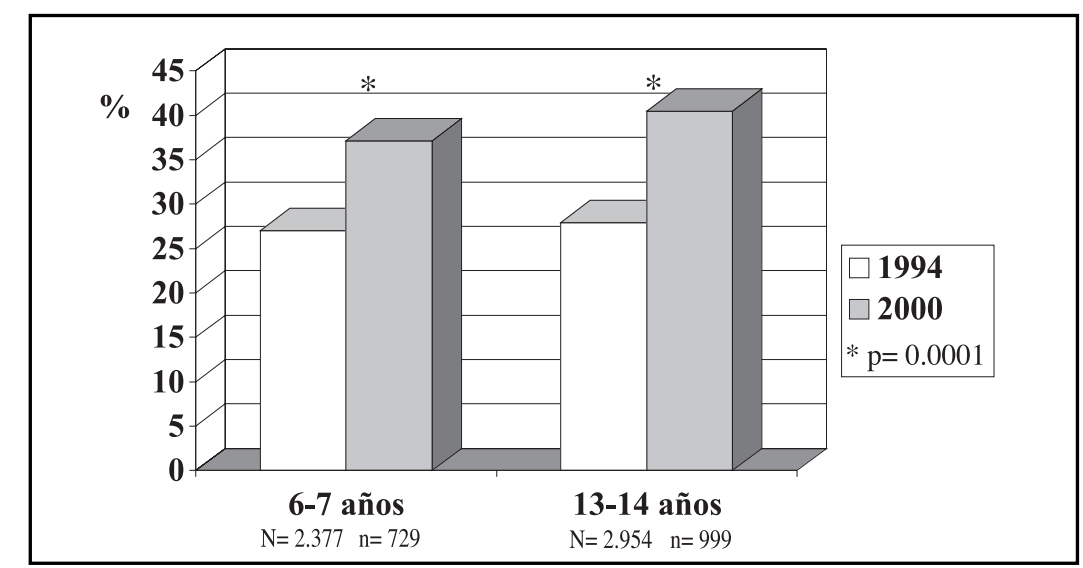

Figura 4. Síntomas de rinitis alérgica últimos 12 meses (SR12). Comparación 1994-2000, área central de Santiago, ambos grupos etáreos.

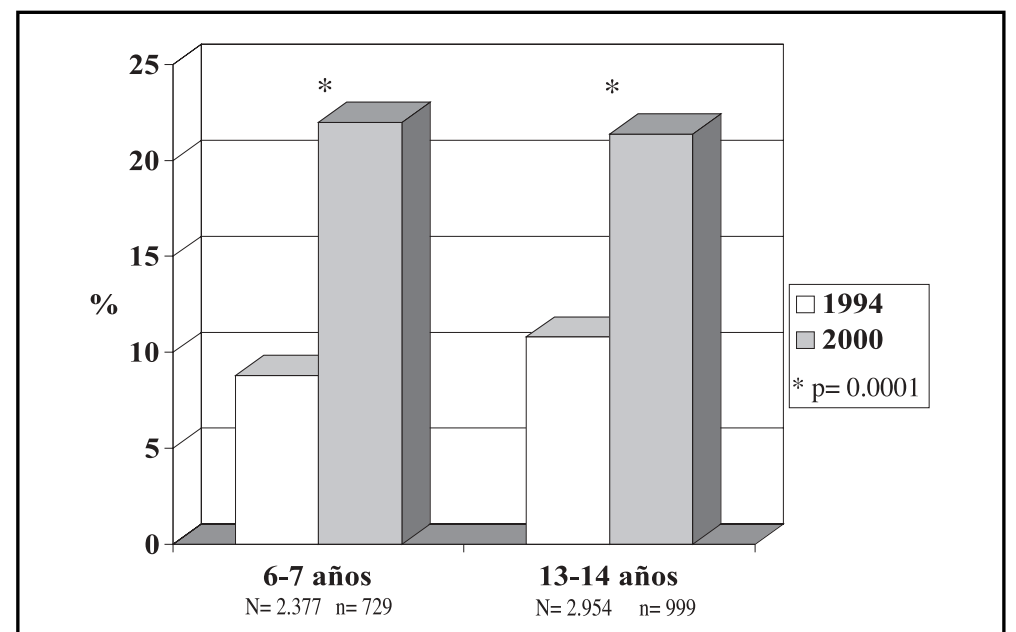

Figura 5. Diagnóstico de rinitis alérgica (DR). Comparación 1994-2000, área central de Santiago, ambos grupos etáreos.

corto período de tiempo (6 años). El diagnóstico de rinitis alérgica se relacionó con un mejor nivel socioeconómico de la población en estudio y con un mejor nivel educacional del padre. Los síntomas de rinitis mostraron relación con nivel socioeconómico medio-bajo del grupo estudiado y con mejor nivel educacional de la madre.

De acuerdo con la información publicada, en la mayoría de los casos, la etiología de la rinitis es la alergia ${ }^{2,4}$, estando relacionada con la introducción precoz de fórmulas lácteas o alimentación sólida, tabaquismo materno durante el primer año de vida, exposición a alergenos intra o extradomi- ciliarios ${ }^{4,12,13,19}$. Además influirían en su desarrollo un mejor nivel socioeconómico, cambios en la dieta, menor tamaño del grupo familiar ${ }^{16-18,20,21}$. El hecho de vivir en zona rural sería un factor protector para el desarrollo de enfermedades alérgicas $^{22-24}$. Los síntomas de rinitis se iniciarían antes de los 20 años en $80 \%$ de los casos, siendo más precoz su aparición mientras mayor historia familiar de alergia exista ${ }^{4,25}$.

Los estudios de prevalencia de esta enfermedad muestran cifras entre 0,5 y $42 \% 11-14,25,26$, lo que depende especialmente de la edad y forma como se realiza el diagnóstico (definición operacional de 
la patología y diagnóstico establecido por médico, realización de cuestionario escrito o vía telefónica). Se debe considerar, además, el grado de percepción de la enfermedad ${ }^{5,7}$. En Chile, en los 4 centros estudiados el año 1994, las cifras variaron entre 8 y $17 \% 6$. El cuestionario ISAAC es fácilmente aplicable, reproducible y tiene alta especificidad para la detección de niños con atopia, al relacionar sus respuestas con pruebas cutáneas; es, por lo tanto, útil para discriminar entre niños con y sin rinitis alérgica $^{7,8}$. Su valor predictivo positivo para atopia, en niños con síntomas, fue de $63 \%$ cuando referían rinorrea acompañado de síntomas oculares y de $67 \%$ cuando los síntomas aparecían durante períodos de polinización ${ }^{23,27}$. Redline y cols $^{28}$ proponen la aplicación de un cuestionario más detallado, que detecta la presencia de rinitis alérgica con una sensibilidad de $83 \%$ y especificidad de $61 \%^{28}$. En estudios no colaborativos, la mayor prevalencia en la población pediátrica está descrita por Wright y cols, siendo ésta de $42 \%$ a los 6 años de vida ${ }^{25}$. Es conocido el aumento en la prevalencia de rinitis en los últimos años, lo que podría estar determinado por la industrialización y los cambios en el estilo de vida: mayor polución ambiental, mejoría de las condiciones habitacionales, mejoría de la higiene, cambios en los hábitos dietarios ${ }^{11-15,29,30}$. Von Mutius y cols describieron una prevalencia de fiebre de heno de 8,6\% en niños de 9 a 11 años residentes en Alemania Occidental, en comparación con una cifra de $3,4 \%$ en los residentes en Alemania Oriental en su estudio realizado entre 1989 y $1992^{14}$. Luego refieren un aumento significativo en Alemania Oriental: 2,3\% en 1991-92 con respecto a 5,1\% en 1995-6, encontrándose relación con un aumento del uso de piso alfombrado, tabaquismo pasivo, humedad intradomiciliaria y presencia de mascotas ${ }^{11}$. Existen otros estudios que sugieren que el aumento de la morbilidad y mortalidad respiratoria se relaciona con la exposición crónica a poluyentes fotoquímicos y material particulado. Calderón y cols describieron, en niños asintomáticos residentes en una zona de alta contaminación ambiental de Nuevo Méjico, una alta incidencia de síntomas nasales, lo que se corroboró con el examen nasal que mostraba signos de daño epitelial ${ }^{31}$. Burr y cols han propuesto que la polución ambiental presente en áreas industriales potenciaría los efectos de los aeroalergenos ${ }^{24}$.
En nuestro estudio observamos que en un corto período de tiempo, SR, SR12 y DR aumentaron en forma significativa, en ambos grupos etarios analizados, sin diferencia por sexo. Vanna $\mathrm{y}$ cols $^{7}$ encontraron una mayor prevalencia en adolescentes mujeres y en hombres de 6-7 años, y Maziak y cols ${ }^{15}$ en niñas, sin tener una explicación clara para estos hallazgos. En nuestro estudio se observó una mayor prevalencia de SR en hogares con cubrepiso o alfombra, y mayor prevalencia de DR cuando había fumadores y calefacción a gas en el hogar. La asociación con la presencia de tabaquismo intradomiciliario y combustibles inusuales había sido descrita por Burr y $\operatorname{cols}^{26}$ y von Mutius y cols ${ }^{11}$.

Encontramos que el nivel socioeconómico se asoció con mayor prevalencia de SR y SR12, lo que podría deberse a confusión de estos síntomas con episodios de resfrío, aunque en el cuestionario se solicita con claridad excluir síntomas relacionados con esta patología. No podemos descartar la posibilidad de sobrediagnóstico basándose en estas dos variables, aunque en los datos del año 1994, las cifras ya eran elevadas, pudiendo observarse quizás el mismo efecto descrito al responder los cuestionarios. Este fenómeno ya había sido descrito por Bousquet ${ }^{8}$, y no concuerda con la alta especificidad del cuestionario ISAAC. La mayor prevalencia de DR en el nivel socioeconómico alto podría explicarse por un mejor acceso a atención de salud y, en el mismo sentido, a un subdiagnóstico en el nivel socioeconómico bajo, por limitación en la cobertura para atención médica especializada. Otras posibles explicaciones serían un mayor conocimiento de esta enfermedad por parte de profesionales de la salud y sus padres, o mayor conciencia de su prevalencia. Existen algunos estudios en los que se relaciona la presencia de atopia y síntomas alérgicos con el nivel socioeconómico: Williams y cols describieron una mayor prevalencia de fiebre de heno en niños de mejor nivel socioeconómi$\mathrm{co}^{32}$, Shaheen y cols encuentran mayor grado de reactividad cutánea a alergenos inhalantes en niños cuyas madres poseen mayor nivel educacional y en familias en las cuales 20 más miembros tienen empleo ${ }^{33}$.

Una limitación relativa de nuestro estudio es su realización el último trimestre del año, durante el período de polinización, lo que podría haber 
determinado mayor probabilidad de reportar síntomas riníticos. Además, el diagnóstico de rinitis es culturalmente conocido en nuestra población, lo que hace posible confundirlo con condiciones de síntomas similares. El estudio siguió una metodología similar a la desarrollada en el estudio ISAAC de fase I (1994), incluyendo la aplicación de terreno, codificación y manejo de las variables ${ }^{6}$.

En conclusión, nuestro estudio confirma que en nuestro país, al igual que en países desarrolla-

\section{REFERENCIAS}

1. BERGER WE. Overview of allergic rhinitis. Annals of Allergy, Asthma and Inmunology 2003; 90: 7-12.

2. O'CONNELL EJ. The burden of atopy and asthma in children. Allergy 2004; 59 (suppl 78): 7-11.

3. Johansson SGO, Hourihane JO'B, Bousquet J, Bruijnzeel-Koomen C, Dreborg S et al. A revised nomenclature for allergy. A EAACI position statement from the EAACI nomenclature task force. Allergy 2001; 56: 813-24.

4. SKoneR DP. Allergic rhinitis: Definition, epidemiology, pathophysiology, detection, and diagnosis. J Allergy Clin Inmunol 2001; 108: S2-8.

5. Asher MI, Keil U, Anderson HR, Beasley R, Crane J, MARTínEZ F ET AL. International study of asthma and allergies in childhood (ISAAC): rationale and methods. Eur Respir J 1995; 8: 483-91.

6. The International Study of Asthma and Aluergies in Childhood (ISAAC). Steering Committee. Worldwide variation in prevalence of symptoms of asthma, allergic rhinoconjuntivitis, and atopic eczema: ISAAC. Lancet 1998; 351: 1225-32.

7. Vanna AT, Yamada E, ArRudalk, Naspitz CK, SolÉ D. International Study of Asthma and Allergies in Childhood: validation of the rhinitis symptom questionnaire and prevalence of rhinitis in school children in Sao Paulo, Brazil. Pediatr Allergy Inmunol 2001; 12: 95-101.

8. Bousquet and the Aria Workshop Group. Introduction and Classification. J Allergy Clin Inmunol 2001; 108: S148-61.

9. Mallol J, Cortez E, Amarales L, Sánchez I, Calvo M, Sото S y cols. Prevalencia del asma en escolares chilenos. Estudio descriptivo de 24.470 niños. ISAAC-Chile. Rev Méd Chile 2000; 128: 279-85.

10. Navarro $\mathrm{H}$, Valdivia G, Caussade S, Pérez E, Aquevedo A y cols. Relación entre rinitis alérgica dos, la prevalencia de síntomas de rinitis ha aumentado en forma considerable y en un corto período de tiempo. Está aún por identificar y confirmar cuáles serían los factores desencadenantes de este fenómeno, en especial variables ambientales determinadas por un mejor estándar de vida. Junto a lo anterior, este estudio señala que posiblemente el mejor acceso a atención en salud es un factor importante para su adecuado diagnóstico.

y asma bronquial en escolares chilenos. Rev Chil Enf Respir 2002; 18: 293.

11. Von Mutius E, Weiland SK, Fritzsch C, Duhme H, KeIL U. Increasing prevalence of hay fever and atopy among children in Leipzig, East Germany. Lancet 1998; 351: 862-6.

12. VaLdivia G. Asma bronquial y enfermedades atópicas como problema emergente en salud pública: nuevas hipótesis etiológicas. La experiencia de sociedades desarrolladas. Rev Méd Chile 2000; 128: 339-46.

13. Weiland SK, Von Mutius E, Hirsch T, Duhme H, FRITZsch C, Werner B et al. Prevalence of respiratory and atopic disorders among children in the East and West of Germany five years after unification. Eur Respir J 1999; 14: 862-70.

14. Von Mutius E, Martínez FD, Fritzsch C, Nicolai T, Roell G, Thiemann H. Prevalence of asthma and attopy in two areas of West and East Germany. Am J Respir Crit Care Med 1994; 149: 358-64.

15. Maziak W, Behrens T, Brasky TM, Duhme H, Rzenah P, Weiland S et al. Are asthma and allergies in children and adolescents increasing? Results from ISAAC phase I and phase II surveys in Munster, Germany. Allergy 2003; 58: 572-9.

16. Eluwood P, Asher Mi, Bjorksten B, Burr M, Pearce $\mathrm{N}$, Robertson CF and the ISAAC Phase One Study Group. Diet and asthma, allergic rhinoconjunctivitis and atopic eczema symptom prevalence: an ecological analysis of the International Study of Asthma and allergies in childhood (ISAAC) data. Eur Respir J 2001; 17: 436-43.

17. Heinrich J, Holscher B, Bolte G, Winkier G. Allergic sensitization and diet: ecological analysis in selected European cities. Eur Respir J 2001; 17: 395-402.

18. McKeever TM, Lewis SA, Smith C, Cowns J, Heatue H, FrISChER M ET AL. Siblings, multiple births, and 
the incidence of allergic disease: a birth cohort study using the West Midlands general practice research database. Thorax 2001; 56: 758-62.

19. SLY M. Changing prevalence of allergic rhinitis and asthma. Ann Allergy 1999; 82: 233-52.

20. Jarvis D, Chinn S, Luczynska C, Burney P. The association of family size with atopy and atopic disease. Clin Exp Allergy 1997; 27: 240-5.

21. Ponsonby AL, Couper D, Dwyer T, Carmichael A. Cross sectional study of the relation between sibling number and asthma, hay fever and eczema. Arch Dis Child 1998; 79: 328-33.

22. Holscher B, Frye C, Wichmann HE, Heinrich J. Exposure to pets and allergies in children. Pediatr Allergy Inmunol 2002; 13: 334-41.

23. Riedler J, Braun-Fahrlander C, Eder W, Scheuer M, WASER M, Maisch S ET Al. Exposure to farming in early life and development of asthma and allergy: a cross-sectional survey. Lancet 2001; 358: 1129-33.

24. Burr ML, Emberlin JC, Treu R, Cheng S, Pearce NE. Pollen counts in relation to the prevalence of allergic rhinoconjuntivitis, asthma and atopic eczema in the International Study of Asthma and Allergies in Childhood (ISAAC). Clin Exp Allergy 2003; 33: 1675-80.

25. Wright AL, Holberg CJ, Martínez FD, Halonen M, Morgan W, TAussig M. Epidemiology of physiciandiagnosed allergic rhinitis in childhood. Pediatrics 1994; 94: 895-901.
26. Burr ML, Anderson HR, Austin JB, Harkins LS, Kaur B, Strachan DP et al. Respiratory symptoms and home environment in children: a national survey. Thorax 1999; 54: 27-32.

27. Kulig M, Kiettke U, Wahn V, Forster J, Bauer CP, WAHN U ET AL. Development of seasonal allergic rhinitis during the first 7 years of life. J Allergy Clin Inmunol 2000; 106: 832-9.

28. Redune S, Larkin EK, Kerscman C, Berger M, SIMINOFF L. Development and validation of school based asthma and allergy screening instruments for parents and students. Ann Allergy Asthma Inmunol 2003; 90: 516-28.

29. Ninan T, Russell G. Respiratory symptoms and atopy in Aberdeen school children: evidence from two surveys 25 years apart. BMJ 1992; 304: 873-5.

30. LeE S, Wong W, LaU Y. Increasing prevalence of allergic rhinitis but not asthma among children in Hong Kong from 1995 to 2001 (Phase 3 International Study of Asthma and Allergies in Childhood). Pediatr Allergy Inmunol 2004; 15: 72-8.

31. Calderón-GarcidueÑas L, Mora-Tiscareño A, Fordham LA, Valencia-Salazar G, Chung ChJ, RodríguezAlcaraz A et aL. Respiratory damage in children exposed to urban pollution. Pediatr Pulmonol 2003; 36: 148-61.

32. Wimams H, Strachan D, Hay R. Childhood eczema: disease of the advantaged? BMJ 1994; 308: 1132-5.

33. Shaheen SO, Aaby P, Hall AJ, Barker DJP, Heyes CB, ShIEL AW et al. Measles and atopy in GuineaBissau. Lancet 1996; 347: 1792-6.

\section{Agradecimientos}

Al Dr. Javier Mallol, coordinador de ISAAC en SudAmérica, por su colaboración para la realización de esta investigación.

A los Sres. Rectores, Inspectores de los siguientes establecimientos educacionales su valiosa colaboración para la realización de este estudio: Colegio del Verbo Divino, SSCC Manquehue, San Benito, San Pedro Nolasco, SSCC Apoquindo, Scuola Italiana, Compañía de María Apoquindo, SEK, Bradford School, Dunalastair, Redland School, La Maisonnette, San Ignacio El Bosque, Lo Castillo, Wenlock School, British High School, Francisco Olea, Guillermo Matta, INSUCO, Isaura Dinator de Guzmán, Benjamín Vicuña Mackenna, Elvira Errázuriz, Miguel de Cervantes y Saavedra, Cambridge School, Blas Cañas, Francisco Arriarán, Cadete Arturo Prat Chacón, Liceo № 1. 